Makarova A. O, Buyadzhi A. A. and Dubrovsky O. V.

\author{
Odesa State Environmental University, L’vovskaya str.15, Odesa-9, 65016, Ukraine \\ e-mail: buyadzhiaa@gmail.com
}

\title{
NUMERICAL STUDYING ENERGY PARAMETERS OF MULTIELECTRON ATOM IN A MAGNETIC FIELD: HELIUM
}

\begin{abstract}
There are presented the results of calculating the energies of the helium atom energy in a homogeneous magnetic field on the basis of the new numerical quantum-mechanical approach. The approach is based on the numerical difference solution of the Schrödinger equation, the model potential method and the operator perturbation theory formalism. The obtained results on energy of the helium atom in dependence upon the magnetic field strength are compared with available theoretical results, obtained on the basis of alternative numerical Hartree-Fock and diagonalization methods.
\end{abstract}

\section{Introduction}

The fundamentals of the quantum theory of the Zeeman effect are described in detail in well-known monographs and in courses on quantum mechanics and theoretical spectroscopy. At present time, interest in these effects shifted to the field of applied research (e.g. [128]). An important problem is studying the energy characteristics of multielectron atoms in a strong and superstrong magnetic field. At the same time, it was found that many of the results of the theory are either of little use for specific calculations, or even incomplete until recently. Further development of the theory is mainly associated with studies of atomic spectra in a sufficiently strong field B and for highly excited states with $n \gg 1$. As indicated in the introduction, interest in these problems is associated with a wide range of applications, including: absorption spectra excitons in a magnetic field, the structure of atoms in superstrong magnetic fields on the surface of neutron stars, the splitting and broadening of atomic spectral lines in electric and magnetic fields of plasma, the structure of radio lines emitted by excited atoms in the interstellar medium, etc.

Among modern methods for describing atomic spectroscopy in a magnetic field, a series of papers [4-18] should be distinguished, where perturbation theory methods, various schemes, and algorithms have been developed based on the numerical solution of the Schrödinger equation in the Hartree-Fock and other approximations. Based on them, it was possible to obtain a lot of useful numerical data re- garding the energies of various states of a number of many-electron atoms at various magnetic field intensities. The imposition of a magnetic field on an atomic system leads to an additional restriction of the electron motion across the field, and with a further increase in the field strength - to a sharp decrease in the transverse motion of the electron and, as a consequence, to the transformation of the three-dimensional potential well of the atom into a one-dimensional one. The consequence of this can be a strong change in the energy spectrum of the atom. For the simplest spinless one-electron (hydrogen) atom, the perturbation Hamiltonian $\mathrm{V}$, caused by the interaction of the orbital momentum $I$ of the electron with the field B, has the form $V=-\mu_{B} I B$. To determine the energy eigenvalues, i.e., diagonalization of the perturbation $\mathrm{V}$, it suffices to choose the wave functions corresponding to a certain projection I onto the direction B. Usually, as such functions, we choose spherical wave functions corresponding to certain values of the total moment $l^{2}=l(l+l)$ and its projection $l_{z}=\hbar m$.

For a hydrogen atom, the Zeeman sublevels corresponding to a certain $m$ remain degenerate in the quantum number 1 . This specificity of the Coulomb degeneracy is also manifested in the fact that as the diagonalizing $V$ wave functions, one can choose parabolic wave functions $\Psi_{n_{1}, n_{2}}$, with the $\mathrm{Oz}$ axis along the field $\mathrm{B}$. These states, by virtue of the relation $n_{1}+n_{2}+|m|=n-1$ for a given $m$, remain degenerate in the values of $n_{1}, n_{2}$ corresponding 
to their equal $\operatorname{sum}_{n_{1}+n_{2} . .}$ Thus, the Zeeman component of the hydrogen level is characterized not by one, but, generally speaking, by several wave functions.

In this paper we shortly present the results of calculating the energies of the helium atom energy in a homogeneous magnetic field on the basis of the new numerical quantummechanical approach $[1,12]$. The approach is based on the numerical difference solution of the Schrödinger equation, the model potential method and the operator perturbation theory formalism.

\section{Theoretical approach}

According to the Ref [10], the Hamiltonian of he helium atom in a magnetic field $B$ differs from the operator of the hydrogen atom by the presence of the Coulomb interaction operator, which naturally aggravates the problem of separation of variables in the Schrödinger equation. Introducing a cylindrical coordinate system $(\rho, \varphi, z)$, with the axis $\mathrm{Oz} \| \mathrm{B}$ and taking into account that the dependence of the wave function on the rotation angle $\varphi$ around the $\mathrm{z}$ axis is trivial:

$$
\Psi \sim e^{i M \varphi} \psi(\rho, z)
$$

one should write the Schrödinger equation for the one-electron function of an atomic system (atomic units are used here $e=h=m=1$ ) as:

$$
\begin{aligned}
& {\left[\frac{\partial^{2}}{\partial \rho^{2}}+\frac{1}{\rho} \frac{\partial}{\partial \rho}+\frac{\partial^{2}}{\partial z^{2}}-\frac{M^{2}}{\rho^{2}}-4 \gamma^{2} \rho^{2}+\right.} \\
& \left.+\frac{4}{r}+V_{c}(r)+\left(\frac{E}{R_{y}}-\gamma M\right)\right] \Psi(\rho, z)=0
\end{aligned}
$$

where $V_{c}(r)$ is the potential that describes the effect of all other electrons on the given one. Naturally, it is absent for the hydrogen atom. As the potential Vc, we use the Green-Miller like potential (c.g.[2]), which approximates the Hartree potential quite accurately.

The required parameters, as a rule, are selected from the condition of the best fitting of the experimental values of the energy levels of free atoms (c.g. [2]).
To take into account the exchange corrections, the exchange potential was taken in the simplest Slater approximation and added to potential (3) [19].

The two-dimensional equation (2) is naturally not solved analytically in a general form. The terms appearing in it: the potential of the Coulomb interaction, which contains $r=\left(\rho^{2}+z^{2}\right)^{1 / 2}$, potential V $\quad\left[\left(\rho^{2}+z^{2}\right)^{1 / 2}\right]$ prevents the separation of variables. One could rewrite the Schrödinger equation as follows:

$$
H \psi(\rho, z)=E \psi(\rho, z)
$$

$$
\begin{gathered}
H=-1 / 2\left(\partial^{2} / \partial \rho^{2}+1 / \rho \partial / \partial \rho+\right. \\
\left.+\partial^{2} / \partial z^{2}-m^{2} / \rho^{2}\right), V(\rho, z), \\
V(\rho, z)=-\left(\rho^{2}+z^{2}\right)^{-1 / 2}+\left(N_{c}-1\right) \\
\Omega\left(\rho^{2}+z^{2}\right)^{-1 / 2}+1 / 8 \gamma^{2} \rho^{2}+\gamma m / 2,
\end{gathered}
$$

The potential $1 / 8 \gamma^{2} \rho^{2}$ limits the movement in the direction perpendicular to the field direction. Similarly, in the region $\gamma \gg 1$, the motion of an electron across a magnetic field is determined by the size of its cyclotron orbit, $\lambda=(\hbar c / e M)^{1 / 2}$ and along the field by a modified Coulomb interaction, which takes into account the non-Coulomb character of the potential field in which an electron moves in a many-electron atom [18].

Note that calculations of multielectron atomic systems with introduced potentials are quite well known in the literature (see [2,1820]); moreover, computational schemes based on them have been tested several times and tested for a number of atoms in the free state. The Green-Miller like potential (c.g.[2]), was successfully used in calculating the energies and forces of atomic oscillators of the 1st period of the periodic table (see review in [21]).

For solution of the Schrödinger equation with hamiltonian equations (7) we constructed the finite differences scheme which is in some aspects similar to method [2]. An infinite region is exchanged by a rectangular region: $0<\rho<L_{\rho}, 0<\mathrm{z}<L_{z}$. It has sufficiently large size; inside it a rectangular uniform grid with 
steps $h_{\rho}, h_{z}$ was constructed. The external boundary condition, as usually, is:

$$
(\partial \Psi / \partial n)_{r}=0
$$

The knowledge of the asymptotic behaviour of wave function in the infinity allows to get numeral estimates for $L_{\rho}, L_{z}$. A wave function has an asymptotic of the kind as: exp[-($2 E)^{1 / 2} r$, where $(-E)$ is the ionization energy from stationary state to lowest Landau level. Then $L$ is estimated as $L \sim 9(-2 E)^{-1 / 2}$. The more exact estimate is found empirically. The threepoint symmetric differences scheme is used for second derivative on $\mathrm{z}$. The derivatives on $\rho$ are approximated by $(2 m+1)$-point symmetric differences scheme with the use of the Lagrange interpolation formula differentiation. To calculate the values of the width $\mathrm{G}$ for resonances in atomic spectra in a magnetic field one can use the modified operator perturbation theory method (see details in Ref.[12,13]).. Other calculation details can be found in Refs. $[2,19-21]$.

\section{Illustration results and conclusion}

As illustration, below we present the data on energies of the electronic excited and ground state of the helium atom in dependence upon the magnetic field strength (parameter $\gamma$ ) and compared with available theoretical results, obtained on the basis of alternative methods.

Parameter $\gamma$ varies within: $\gamma=B / B o=0.00-10$, where $B_{0}=m^{2} e^{3} c / h^{3} Z^{3}$.

In Table 1 there are listed the energies of the ground state of the helium atom in dependence upon the parameter $\gamma$. For the helium atom there are available the results of calculations for the ground state within other theoretical methods. In particular, the Hartree-Fock (HF) calculation results are in the Refs. [6,7].

As the ground state analysis shows, in whole our data are corresponding to the alternative HF results, however, indeed, they lie a little lower for a weak field regime and more substantially lower in the intermediate regime of the magnetic parameter.
Table 1

The energies (in atomic units) of the ground state of the helium atom as a function of the magnetic field parameter $\gamma$

\begin{tabular}{|c|c|c|c|c|}
\hline Method & $\gamma=0.5$ & $\gamma=1$ & $\gamma=2$ & $\gamma=5$ \\
\hline$[15]$ & - & $-2,400$ & - & - \\
{$[12\}$} & $-2,700$ & $-3,027$ & $-3,520$ & $-4,624$ \\
{$[13]$} & $-2,616$ & $-2,960$ & $-3,502$ & $-4,617$ \\
Our & $-2,700$ & $-3,027$ & $-3,520$ & $-4,624$ \\
\hline Method & $\gamma=10$ & $\gamma=20$ & $\gamma=50$ & $\gamma=100$ \\
\hline$[12]$ & $-5,860$ & $-7,450$ & $-10,288$ & $-13,343$ \\
{$[13]$} & $-5,829$ & $-7,428$ & $-10,264$ & $-13,077$ \\
Our & $-5,860$ & $-7,450$ & $-10,288$ & $-13,343$ \\
\hline Method & $\gamma=200$ & $\gamma=500$ & $\gamma=1000$ & $\gamma=10000$ \\
\hline$[12]$ & $-16,602$ & $-22,471$ & $-28,342$ & $-56,623$ \\
{$[13]$} & $-16,579$ & $-22,467$ & $-28,032$ & $-55,151$ \\
Our & $-16,602$ & $-22,471$ & $-28,342$ & $-56,623$ \\
\hline
\end{tabular}

The difference between the listed data can be explained by the partial account of electron correlation corrections, which is absent in the HF calculation.

\section{References}

1. Makarova, A., Buyadzhi, A., Dubrovsky, O. Spectroscopy and dynamics of multielectron atom in a magnetic field: New approach. Photoelectronics. 2019, 28, 19-23.

2. Khetselius, O.Yu. Hyperfine structure of atomic spectra. Astroprint: Odessa, 2008.

3. Khetselius, O.Yu. Optimized relativistic many-body perturbation theory calculation of wavelengths and oscillator strengths for Li-like multicharged ions. Adv. Quant. Chem. 2019, 78, 223-251.

4. Glushkov, A.V. Relativistic Quantum theory. Quantum mechanics of atomic systems. Astroprint: Odessa, 2008.

5. Khetselius, O.Yu. Quantum structure of electroweak interaction in heavy finite Fermi-systems. Astroprint: Odessa, 2011.

6. Glushkov, A.V. Relativistic and correlation effects in spectra of atomic systems. Astroprint: Odessa, 2006 .

7. Ivanova, E., Glushkov, A. Theoretical investigation of spectra of multicharged ions of F-like and Ne-like isoelectronic sequences. J. Quant. Spectr. and Rad. Tr. 1986, 36(2), 127-145. 
8. Ivanova, E.P., Ivanov, L.N., Glushkov, A., Kramida, A. High order corrections in the relativistic perturbation theory with the model zeroth approximation, Mg-Like and Ne-Like Ions. Phys. Scripta 1985, 32, 513522.

9. Ignatenko, A., Svinarenko A., Prepelitsa, G.P., Perelygina, T.B. Optical bi-stability effect for multi-photon absorption in atomic ensembles in a strong laser field. Photoelectronics. 2009, 18, 103-105.

10. Rao,J., Liu, W., Li, B. Theoretical complex Stark energies of hydrogen by a complexscaling plus B-spline approach. Phys. Rev. A. 1994, 50, 1916-1919.

11. Rao, J.; Li, B. Resonances of hydrogen atom in strong parallel magnetic and electric fields. Phys.Rev.A. 1995, 51, 4526

12. Glushkov, A.V. Atom in an electromagnetic field. KNT: Kiev, 2005.

13. Ivanov M.V., Schnelcher P. Ground States of $\mathrm{H}, \ldots, \mathrm{Ne}$ and their singly positive ions in strong magnetic fields: the high-field regime. Phys.Rev.A.-2000., 61, 022505.

14. Jones, M., Ortiz, G., Ceperley, D. HartreeFock studies of atoms in strong magnetic fields. Phys. Rev. A. 1996, 54, 219-231.

15. Gadiyak G., Lozovik Yu.E., Mashchenko A., Obrecht M. Hydrogen-like and heliumlike systems in a superstrong magnetic field. Opt. Spectr. 1984, 56 (1), 26-32

16. Khetselius, O., Lopatkin Y., Dubrovskaya, Y., Svinarenko A. Sensing hyperfinestructure, electroweak interaction and parity non-conservation effect in heavy atoms and nuclei: New nuclear-QED approach. Sensor Electr. and Microsyst. Techn. 2010, 7(2), 11-19.

17. Glushkov, A., Malinovskaya, S, Ambrosov S., Shpinareva I, Troitskaya O. Resonances in quantum systems in strong external fields: consistent quantum approach. $J$. Techn.Phys. 1997, 38(2), 215-218.

18. Ambrosov S., Ignatenko V., Korchevsky D., Kozlovskaya V. Sensing stochasticity of atomic systems in crossed electric and magnetic fields by analysis of level statistics for continuous energy spectra. Sensor Electr. and Microsyst. Techn. 2005, Issue 2, 19-23.

19. Glushkov, A.; Ambrosov, S.; Ignatenko, A. Non-hydrogenic atoms and Wannier-
Mott excitons in a DC electric field: Photoionization, Stark effect, Resonances in ionization continuum and stochasticity. Photoelectronics, 2001, 10, 103-106.

20. Glushkov A.V.; Ivanov, L.N. DC strongfield Stark effect: consistent quantummechanical approach. J. Phys. B: At. Mol. Opt. Phys. 1993, 26, L379-386.

21. Rusov V., Glushkov A., Vaschenko V., Korchevsky D., Ignatenko A. Stochastic dynamics of the atomic systems in the crossed electric and magnetic field: the rubidium atom recurrence spectra. Bull.of Kiev Nat. Univ. 2004, N4, 433.

22. Khetselius, O. Relativistic perturbation theory calculation of the hyperfine structure parameters for some heavy-element isotopes. Int. J. Quant.Chem. 2009, 109, 3330-3335.

23. Khetselius, O. Relativistic calculation of the hyperfine structure parameters for heavy elements and laser detection of the heavy isotopes. Phys.Scr. 2009, T135, 014023.

24. Glushkov, A.; Gurskaya, M.; Ignatenko, A.; Smirnov, A.; Serga, I.; Svinarenko, A.; Ternovsky E. Computational code in atomic and nuclear quantum optics: Ad-vanced computing multiphoton resonance parameters for atoms in a strong laser field. $J$. Phys.: Conf. Ser. 2017, 905, 012004.

25. Buyadzhi, V., Zaichko, P., Antoshkina, O., Kulakli, T., Prepelitsa, P., Ternovsky, V., Mansarliysky, V. Computing of radiation parameters for atoms and multicharged ions within relativistic energy approach: Advanced Code. J. Phys.: Conf. Ser. 2017, 905(1), 012003.

26. Svinarenko, A., Glushkov, A., Khetselius, O., Ternovsky,V., Dubrovskaya, Yu., Kuznetsova, A., Buyadzhi, V. Theoretical spectroscopy of rare-earth elements: spectra and autoionization resonances. Rare Earth Element, InTech. 2017, 83-104

27. Glushkov, A., Khetselius, O., Svinarenko A.A., Buyadzhi, V.V., Ternovsky, V., Kuznetsova, A., Bashkarev, P. Relativistic perturbation theory formalism to computing spectra and radiation characteristics: application to heavy element. Recent Studies in Perturbation Theory. InTech. 2017, 131-150. 
28. Glushkov A.V., Khetselius O.Yu., Svinarenko A.A., Buyadzhi V.V., Methods of computational mathematics and mathematical physics. TES: Odessa, 2015
29. Khetselius, O., Gurnitskaya, E., Loboda, A., Vitavetskaya, L. Consistent quantum approach to quarkony energy spectrum and semiconductor superatom and in external electric field Photoelectron. 2008, 17, 127.

PACS 31.15.Ne, 31.10.1z

Makarova A.O, Buyadzhi A.A. and Dubrovsky O.V.

\section{NUMERICAL STUDYING ENERGY PARAMETERS OF MULTIELECTRON ATOM IN A MAGNETIC FIELD: HELIUM}

Summary. There are presented the results of calculating the energies of the helium atom energy in a homogeneous magnetic field on the basis of the new numerical quantum-mechanical approach. The approach is based on the numerical difference solution of the Schrödinger equation, the model potential method and the operator perturbation theory formalism. The obtained results on energy of the helium atom in dependence upon the magnetic field strength are compared with available theoretical results, obtained on the basis of alternative numerical HartreeFock and diagonalization methods.

Key words: atomic system, magnetic field, quantum mechanical approach

PACS 31.15.Ne, 31.10.1z

Макарова А.О., Буяджи А.А., Дубровский О.В.

\section{ЧИСЛЕННОЕ ИССЛЕДОВАНИЕ ЭНЕРГЕТИЧЕСКИХ ПАРАМЕТРОВ МНОГОЭЛЕКТРОННОГО АТОМА В МАГНИТНОМ ПОЛЕ: ГЕЛИЙ}

Резюме. Представлены результаты расчета энергии атома гелия в однородном магнитном поле на основе нового численного квантово-механического подхода. Подход основан на численном конечно--азностном решении уравнения Шредингера, методе модельного потенциала и формализме операторной теории возмущений. Полученные результаты по энергии атома гелия в зависимости от напряженности магнитного поля сравниваются с известными теоретическими результатами, полученными на основе альтернативных численных методов Хартри-Фока и диагонализации.

Ключевые слова: атомная система, магнитное поле, квантово-механический метод 
PACS 31.15.Ne, 31.10.1z

Макарова О.О., Буяджи Г.А., Дубровський О.В.

\section{ЧИСЕЛЬНЕ ВИВЧЕННЯ ЕНЕРГЕТИЧНИХ ПАРАМЕТРІВ БАГАТОЕЛЕКТРОННИХ АТОМІВ В МАГНІТНОМУ ПОЛІ: ГЕЛІЙ}

Резюме. Представлені результати розрахунку енергії атома гелію в магнітному полі на основі нового чисельного квантово-механічного підходу. Підхід заснований на чисельному скінченно-різницевому розв'язанні рівняння Шредінгера, методі модельного потенціалу та формалізмі операторної теорії збурень. Отримані результати по енергії атома гелію в залежності від напруженості магнітного поля порівнюються з відомими теоретичними результатами, отриманими на основі альтернативних чисельних методів Хартрі-Фока і діагоналізації.

Ключові слова: атомна система, магнітне поле, квантово-механічний метод 